\title{
Dental pulp responses to biphasic calcium phosphate cement containing buffer solution in the liquid phase
}

\author{
M. Yoshikawa \& T. Toda \\ Department of Endodontics, Osaka Dental University, Japan
}

\begin{abstract}
We developed a novel biphasic calcium phosphate cement of dibasic calcium phosphate dihydrate and tetracalcium phosphate as a root canal filling material. For endodontic clinical use, the physical properties and tissue responses were estimated. The $\mathrm{pH}$ level ranged from 8.2 to 8.6. Low crystalline hydroxyapatite was recognized by XRD analysis. Mutually connected platy particles and spherical particles were observed by SEM examination. The particles were separated from each other by about $10 \mu \mathrm{m}$. Dental pulp responses to the cement was estimated in mandibular first molar in vivo. Hard tissue was formed in the middle of root canal by placement of the cement on the amputated pulp at the orifice. When the cement was placed on the pulp amputated at the middle in the root canal, cementum-like hard tissue was formed at the apex. Pulp amputation and placement of the cement at the apical portion, mild inflammatory responses to the cement were found in the periapical area. The cement in the alveolar bone defect via the root canal promoted the reconstruction of periapical alveolar bone defect. It is concluded that the cement is suitable for endodontic bioactive material because of hard tissue inductiveness.
\end{abstract}

Keywords: calcium phosphate cement, dental pulp, hard tissue formation.

\section{Introduction}

It is known that dental pulp tissue (pulp) is surrounded by hard tissue of dentine. Moreover the dentine of the coronal portion is covered with enamel, and dentine of the tooth root is covered with cementum. Hard tissue defect caused by injury or dental caries is restored with resin or metals. Pulp is often exposed in the oral environment due to tooth fracture or dental caries. Pulp is immature, and shows 
an embryonic character [1]. Therefore, once the pulp is exposed in the oral environment, the pulp must be extirpated. It is because that the exposed pulp will be infected by bacterial flora in the oral cavity [2] and inflammation easily develops. Subsequently, the hollow canal after pulp extirpation should be obturated hermetically to prevent infection from the oral cavity [3, 4]. Ideal healing after pulp extirpation involves hard tissue deposition in the residual pulp to close the apical root canal [5]. Hard tissue inductivity is also expected of the filling material.

Chow reported biphasic calcium phosphate cement as a biocompatible dental restorative cement paste [6]. This calcium phosphate paste is hardened with water or diluted organic acid. Calcium phosphate cements are reported to show excellent tissue compatibility [7-9] and hard tissue inductivity [10, 11]. We developed novel biphasic calcium phosphate cement. The powder phase of the cement consisted of an equimolar mixture of tetracalcium phosphate and dibasic calcium phosphate-dihydrate as described by Chow. The mixture shows buffer action as a solid phase. For the cement, we selected a buffer solution as the liquid phase. In the setting process, each buffer action of solid and liquid phase contributes to maintain the $\mathrm{pH}$ of the cement about neutral range [12].

We wished to use the cement as a novel endodontic material for sealing of an apical root canal. For endodontic clinical use, the $\mathrm{pH}$ level during the setting process, disintegration ratio, X-ray diffraction (XRD) analysis and micro construction after setting of the cement were examined in this study. And responses of pulp and periapical tissue to the cement were also examined in vivo.

\section{Materials and methods}

\subsection{Preparation of calcium phosphate cement}

Tetracalcium phosphate (TeCP) was prepared by heating a stoichiometric mixture of dibasic calcium phosphate dihydrate (DCPD) and calcium carbonate (Mol ratio of $\mathrm{Ca} / \mathrm{P}=2.0$ ) at $1,400^{\circ} \mathrm{C}$ for 8 hours. DCPD was purchased from Wako Pure Chemical (Osaka, Japan). Both powders were passed through a 32 $\mu \mathrm{m}$-sieve in order to standardize the particle sizes. The sizes of TeCP and DCPD particles were measured with a particle size distribution indicator (9230 HRA X100, Nikkiso Co., Ltd., Tokyo, Japan) by laser diffraction analysis using a unified scatter technique.

Biphasic calcium phosphate cement consisted of an equimolar mixture of DCPD and TeCP. The mixture is abbreviated as TeDCPD in this paper. The TeDCPD was kneaded with a buffered solution containing a low concentration of citric acid. Components of the cement are shown in Table 1. This cement is also abbreviated as n-TeDCPD cement in this paper.

\subsection{Measurement of $\mathrm{pH}$ level during setting process}

The $\mathrm{pH}$ level of $\mathrm{n}$-TeDCPD cement was measured for 120 minutes at 15 -minute intervals after kneading. The cement was kneaded for 1 minute with a 
powder/liquid ratio of $1.2(\mathrm{~g} / \mathrm{ml})$. The $\mathrm{pH}$ level at each time point was measured three times. The cement after kneading was immediately placed on the sensor of a compact type $\mathrm{pH}$ meter (Cardy-II: Horiba, Kyoto, Japan), and the $\mathrm{pH}$ of the cement were measured over time. Significance of differences was evaluated by Student's t-test $(\mathrm{p}<0.005)$.

\subsection{X-ray diffraction (XRD) analysis}

The cements kneaded for 1 minute with a powder/liquid ratio of $1.2(\mathrm{~g} / \mathrm{ml})$ was stored in an incubator at $37 \pm 1{ }^{\circ} \mathrm{C}$ with $99 \%$ relative humidity until XRD analysis. The crystalline phases of the cement in the setting process at 1 hour, 1 day, 3 days and 7 days after kneading were determined by X-ray diffract meter (XRD-6100: SHIMADZU, Kyoto, Japan) using $\mathrm{Cu} \mathrm{K} \alpha$ radiation generated at 40 $\mathrm{kV}$ and $30 \mathrm{~mA}$ and a graphite monochrome meter. The divergence slit was $1^{\circ}$ and receiving slit width was $0.15 \mathrm{~mm}$. The scanning range of the sample was from $10^{\circ}$ to $60^{\circ}$ with a scanning speed of $2^{\circ} / \mathrm{min}$.

\subsection{Micro construction of cement by scanning electric microscopy (SEM)}

After kneading of $\mathrm{n}$-TeDCPD cement with a powder/liquid ratio of $1.2(\mathrm{~g} / \mathrm{ml})$, the cement was kept for 24 hours under a condition of at $37 \pm 1^{\circ} \mathrm{C}$ in $99 \%$ relative humidity in an incubator. Then, the hardened cement was dried at $60^{\circ} \mathrm{C}$ in an incubator. The cement was split and coated with Au-Pt according to the routine methods for SEM. The surface and internal microstructure of the cement were observed with SEM (Hitachi S-2100 A, Hitachi Ltd., Tokyo, Japan).

Table 1: $\quad$ Components of n-TeDCPD cement.

\begin{tabular}{|lc|lc|}
\hline Powder phase & $($ wt \%) & Liquid phase & (wt \%) \\
\hline \multirow{2}{*}{ Tetracalcium phosphate } & \multirow{2}{*}{68.0} & Citric acid & 2.1 \\
\cline { 3 - 4 } & \multirow{2}{*}{$\begin{array}{c}\text { Dibasic calcium } \\
\text { phosphate-dihydrate }\end{array}$} & Dibasic sodium phosphate & 7.2 \\
\cline { 3 - 4 } & \multirow{2}{*}{32.0} & Carboxymethyl cellulose & 3.0 \\
\hline
\end{tabular}

\subsection{Histological examination in the pulp}

To estimate hard tissue formation in dental pulp, mandibular first molar root canals of six-week-old SD male rats were used. This study was performed in the Laboratory Animal Facilities in the Institute of Dental Research, Osaka Dental University, and performed under the "Guidelines for Animal Experimentation at Osaka Dental University". All procedures were approved by the Animal Welfare Committee of Osaka Dental University on Use and Care of Animals. The rats were kept in standard rat cages with free access to dry pellets and water with unrestricted movement at all times.

All operations on the animals were performed under general anesthesia by intraperitoneal injection with sodium pentobarbital (Nembutal, 4mg/100g body 
weight). After establishing aseptic conditions by swabbing teeth and oral mucosa with popidone iodine, access cavities in the mandibular right and left first molars was prepared with an engine driven No.1/2 round bur while dropping sterilized distilled water on the occlusal surface of the tooth during the procedure. Then, coronal pulp was removed. No cement was placed on the residual pulp surface with regard to 20 root canals (Group 1). The n-TeDCPD cement was placed on the residual pulp surface at the root canal orifice in 20 root canals (Group 2). The pulp in 20 root canals of mandibular first molars was amputated at the middle portion (Group 3). The pulp in 20 root canals was extirpated at the apical portion of the root canal (Group 4). The procedures were performed with No. 15 to 30 K-type endodontic files. The n-TeDCPD cement was mildly placed on the surface of residual pulp at each level of amputation without the pulp in Group 1. Moreover, to estimate the promotion of periapical alveolar bone reconstruction by cement, the total pulp to the apical portion in the mesial root canals was extirpated using No. 15 to $30 \mathrm{~K}$-type endodontic files. Then, $2 \mathrm{~mm}$ of a $30 \mathrm{~K}$ type endodontic file tip was pushed into the periapical areas through the apical foramen to injure the periapical alveolar bone of the medial root. As a control, no cement was introduced into the injured periapical areas of 20 roots (Group 5). In the mechanically injured periapical area of 20 medial roots, n-TeDCPD cement was introduced through the access cavity via the prepared root canal using an engine-driven Lenturo spiral (Group 6). All tooth chambers were hermetically sealed with a light curing adhesive resin. The animals were euthanased by excessive dosage of pentobarbital 3 and 5 weeks postoperatively.

At the predetermined endpoint of the experiment, the mandibles were dissected. Following fixation in $10 \%$ buffered formalin solution, the specimens were processed histologically. They were decalcified in $10 \%$ ethylenediamine tetraacetic acid solution, dehydrated in 100\% ethyl alcohol, and embedded in paraffin. Serial sections $6 \mu \mathrm{m}$ thick were cut and stained with hematoxylin and eosin. The sections were observed histologically under an optical microscope.

\section{Results}

\subsection{Preparation of calcium phosphate}

The sizes of TeCP and DCPD particles, measured with a particle size distribution indicator by laser diffraction analysis using unified scatter technique, ranged in size from 0.6 to $44.0 \mu \mathrm{m}$ with a $12 \mu \mathrm{m}$ mean.

\subsection{Measurement of $\mathrm{pH}$ level during setting process}

The $\mathrm{pH}$ level of $\mathrm{n}$-TeDCPD cement during the setting process ranged from approximately 8.2 to 8.6 . The $\mathrm{pH}$ of the cement measured over time was $8.21 \pm$ 0.11 just after kneading, $8.50 \pm 0.10$ at 15 and 45 minutes, $8.54 \pm 0.05$ at 30 minutes, $8.52 \pm 0.06$ at 60 minutes, $8.62 \pm 0.04$ at 75 minutes and $8.51 \pm 0.05$ from 90 to 120 minutes after kneading. The cement showed significant 
differences in $\mathrm{pH}$ on comparison between values just after and 15 to 120 minutes after kneading.

\subsection{XRD analysis}

The phase composition of n-TeDCPD cement were evaluated by XRD 1 hour, 1 day, 3 days and 7 days after kneading. The scanning patterns 1 hour and 3 days after kneading are shown in Fig. 1. One hour after kneading the cement, TeCP, DCPD and hydroxyapatite (HAP) were observed. One day after kneading, peaks indicating TeCP and HAP without DCPD were recognized. TeCP was slightly and HAP was clearly recognized 3 days after kneading. Seven days after kneading, only HAP was recognized. XRD analysis indicated n-TeDCPD cement had converted to HAP with low crystallinity.

a) I (cps)

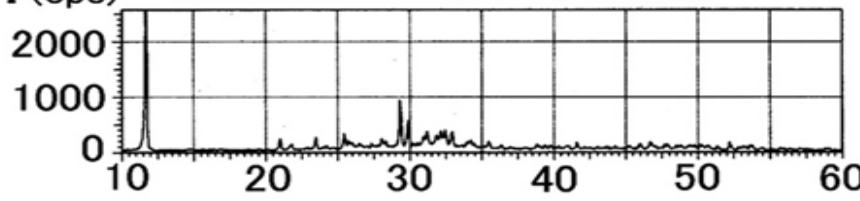

b) I (cps)

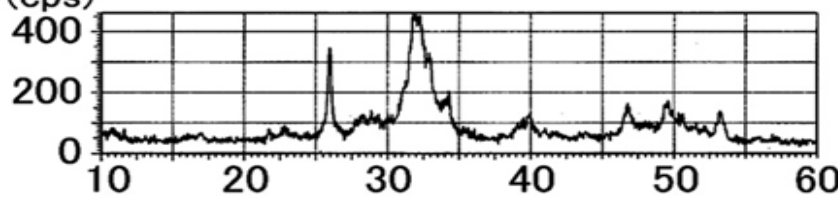

Figure 1: XRD analysis of n-TeDCPD cement a) 1 hour after kneading. b) 3 days after kneading.
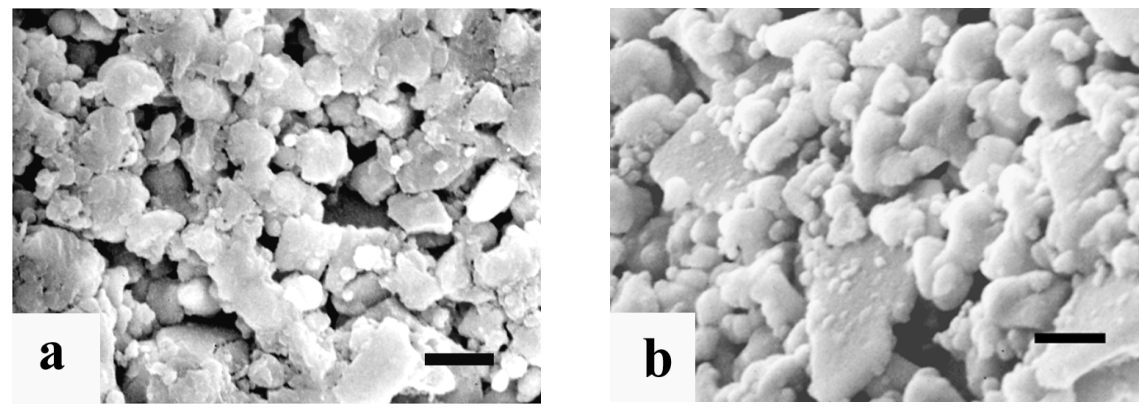

Figure 2: SEM image of n-TeDCPD cement (bur: 10 $\mu \mathrm{m}$ ) a: Surface of the cement, b: Split surface of the cement. 


\subsection{Micro construction of cement by SEM}

Surface and internal construction of n-TeDCPD cement were observed by SEM. Platy and mainly spherical micro particles measuring 2-16 $\mu \mathrm{m}$ along the major axis were observed connecting mutually on the surface of the cement (Fig. 2-a). Many platy particles in measuring approximately $10-25 \mu \mathrm{m}$ along the major axis and a small number of spherical micro particles $1-20 \mu \mathrm{m}$ in diameter were found on the split surface of the cement as shown in Fig. 2-b. The spaces between the particles were usually less than $10 \mu \mathrm{m}$.
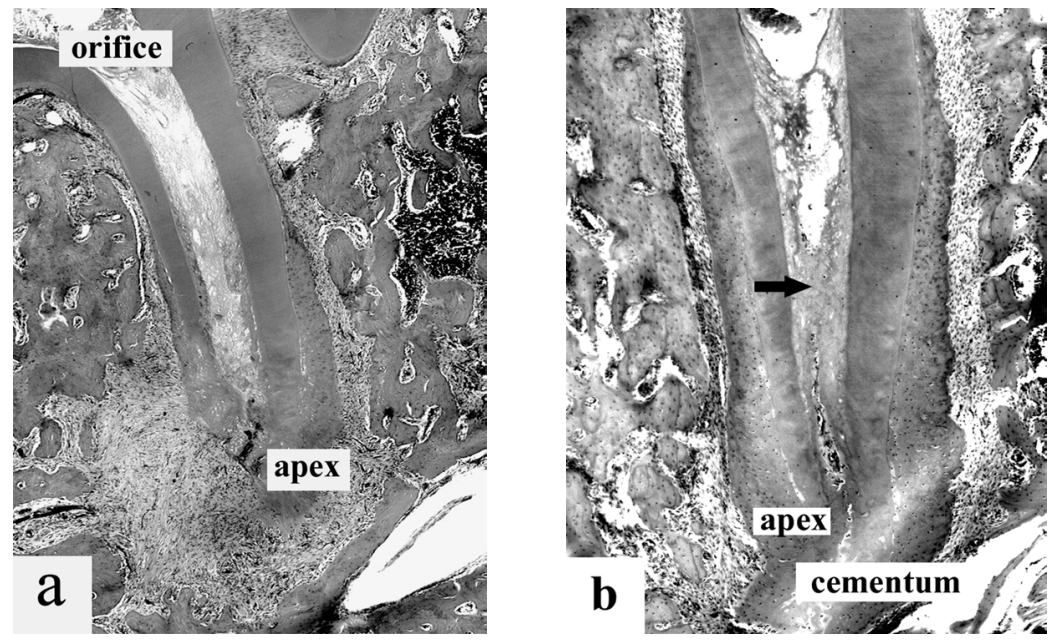

Figure 3: Pulp was amputated at the orifice 5 weeks postoperatively a: No cement was placed on the amputated pulp. $\quad(\times 10), \mathrm{b}: \mathrm{n}-\mathrm{TeCPD}$ cement was placed on the amputated pulp surface. Arrow: Newly formed hard tissue $(\times 17)$.

\subsection{Histological findings on hard tissue deposition in the pulp}

In Group 1, necrosis of the residual pulp near the orifice was observed 3 weeks postoperatively. The pulp fell into necrosis to the apical portion 5 weeks postoperatively (Fig. 3-a). In Group 2, hard tissue deposition on the root canal wall at the middle portion was observed by placement of n-TeDCPD cement (100\%: n=19) 3 weeks postoperatively (Fig. 3-b). The apical portion of the root canal was completely closed with new hard tissue. In Group 3, n-TeDCPD cement induced mild inflammatory reactions in the surface layer of residual pulp 3 weeks postoperatively. As shown in Fig. 4-a, the apical foramen was covered with cementum-like hard tissue 5 weeks postoperatively $(85 \%: n=20)$. After pulp extirpation at the apex and n-TeDCPD cement was filled in the root canal as in Group 4, periapical alveolar bone absorption was conspicuous $(100 \%: n=20) 3$ weeks postoperatively. Granulation tissue was still present in the periapical area 5 weeks postoperatively (Fig. 4-b). Cementum deposition around the apex was 
not seen. In Group 5, reconstruction of defected alveolar bone was not recognized $(n=20) 5$ weeks postoperatively. In Group 6, extruded n-TeDCPD in the periapical area (Fig. 5-a) was resolved and. reconstruction of alveolar bone occurred simultaneously 5 weeks postoperatively (Fig. 5-b).
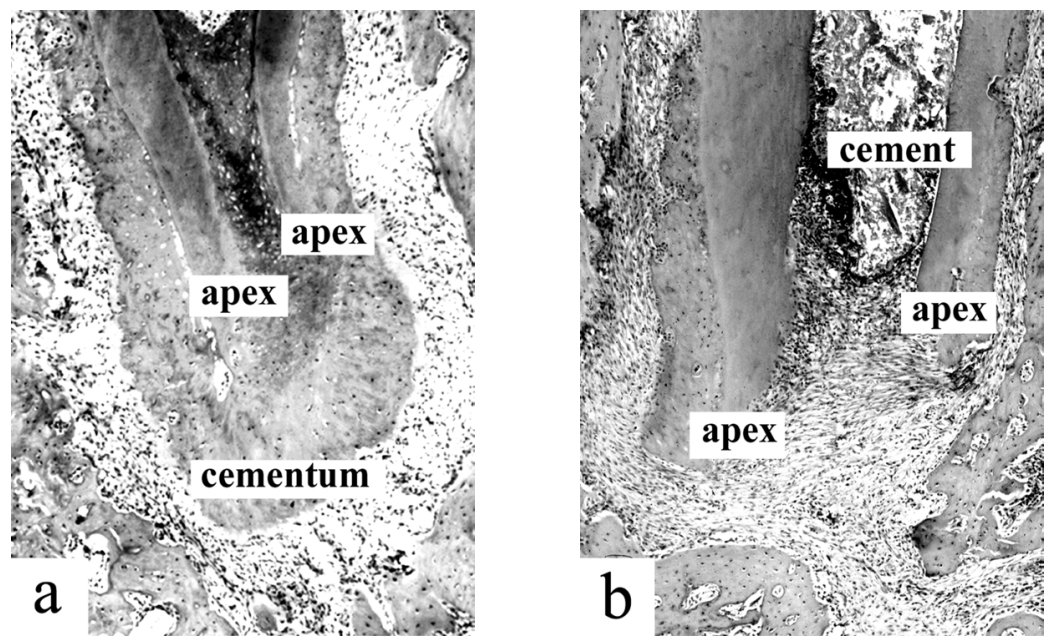

Figure 4: Five weeks postoperatively a: n-TeDCPD cement was placed on the residual pulp surface at the middle of the root canal. $(\times 25)$. b: $n$ TeDCPD cement (cement) was placed on the pulp remnant at the apical root canal. $(\times 20)$.
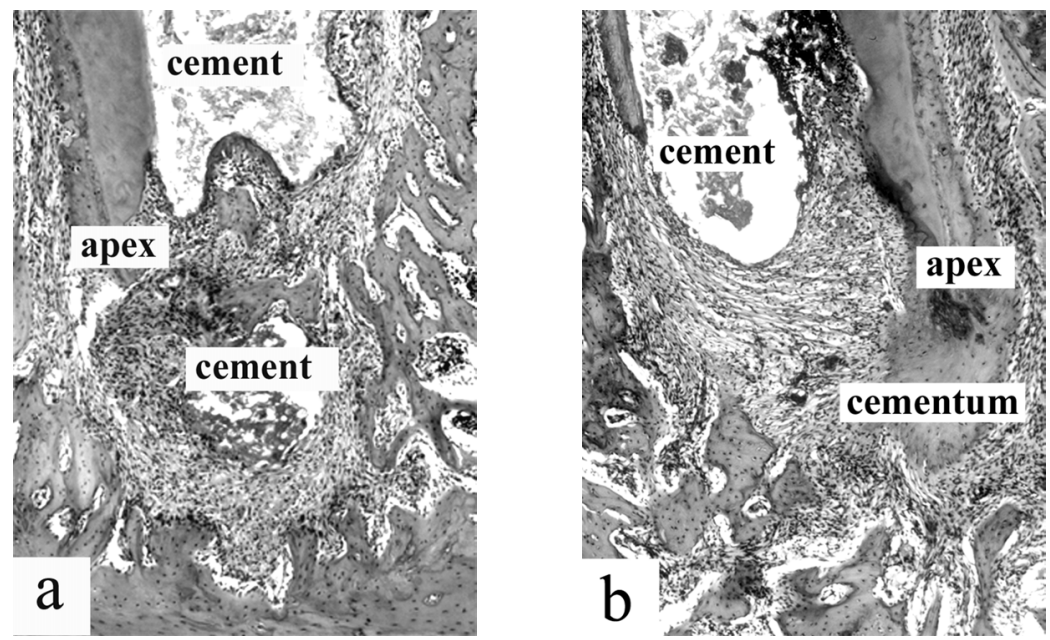

Figure 5: n-TeDCPD cement was extruded in the periapical region after pulp extirpation and root canal preparation. a: 3 weeks postoperatively $(\times 20)$ b: 5 weeks postoperatively $(\times 20)$. 


\section{Discussion}

The powder phase of n-TeDCPD cement tested in this study consisted of equimolar mixture of DCPD and TeCP. The formula of the powder phase was same as that developed by Chow [6]. The biphasic powder phase shows a buffer action during setting reaction [13]. Two-fold concentration of McIlvain's buffer solution was selected for the liquid phase of n-TeDCPD cement. The buffer actions of both the solid and liquid phase of $n$-TeDCPD cement may contribute to maintaining $\mathrm{pH}$ level of the cement in a neutral range during the setting process. The cement, showing a $\mathrm{pH}$ value in a neutral range, may cause less stimulation of the surrounding tissue.

In the early postoperative stage, mild to severe inflammation may occur in the residual pulp. Usually, inflammatory reactions occur after pulp amputation because of surgical irritation [14]. The mechanical stimulation of pulp amputation or extirpation would affect the residual pulp and radicular pulp would become necrotic [15]. This may be because of the histological and embryological immaturity of pulp tissue. Moreover, if fine particles of calcium phosphate cement scattered in the surrounding tissue, the fine particles would induce an accumulation of phagocytes, causing inflammatory responses in the tissue [16]. There are odontoblasts along pulp canal wall and undifferentiated mesenchymal cells in pulp tissue [1]. When there was no n-TeDCPD cement placed over the surface of the amputated residual pulp, necrosis of the residual pulp and devitalization of the pulp cells occurred. This might be because mechanical stimulus after amputation was severe and caused inflammation in the injured residual pulp. Severe inflammation because of mechanical stimulus extended in the residual pulp and deactivation would extend to the apical portion of the root. The nutrition supply from the narrow apical foramen might insufficient for pulp healing. And insufficient nutrition supply should cause deactivation of residual pulp to apical area.

However, n-TeDCPD cement promoted wound healing and induced hard tissue in the root canal wall in this study. There were differences in the responses of hard tissue deposition by n-TeDCPD cement in accordance with the position of pulp amputation. Pulp amputation at the root canal orifice may affect the residual pulp by mechanical stimulus. Odontoblasts are the most differentiated cells in the pulp and form dentine, while undifferentiated pulp cells would be affected by the physiological stimulus. Only the surface layer of the residual pulp was injured by pulp amputation and the stimulus of n-TeDCPD cement in the setting process would physiologically affect activation of cells in the residual pulp and maintaining the pulp normal. When pulp was amputated at the middle portion of the root canal, the volume of residual pulp was limited. Therefore, stimulation by amputation might easily expand throughout the whole area of residual pulp. The odontoblasts and undifferentiated pulp cells in the residual pulp might be deactivated by the stimulations.

It was considered that mesenchymal cells in the periapical tissue differentiated in hard tissue-forming cells and these cells then formed cementumlike hard tissue around the apex. 
Our previous histological studies [14, 17] showed that the length of a sixweek-old SD rat mandibular first molar canal from the cusp of crown to the root apex is approximately $4 \mathrm{~mm}$. Therefore, $2 \mathrm{~mm}$ of the instrument tip was pushed into the periapical area to injure the alveolar bone. In this study, severe damage in the periapical region was found 3 weeks postoperatively. Neither reconstruction of the alveolar bone nor wound healing in periapical tissue occurred only by sealing of the pulp chamber, without n-TeDCPD cement placement in the periapical bone defect.

Generally wound healing begins by proliferation of granulation tissue in defect. As shown in this study, conversion of a granulation tissue to bone takes considerable time. However, when biodegradative calcium phosphate cement fills a bone defect, reconstruction might be promoted because absorption of cement and addition of new bone progress at the same time [17]. It was shown by XRD analysis in this study that TeCP in the n-TeDCPD cement immediately participated in the reaction during the setting process and converted to low crystalline HAP. The n-TeDCPD cement may be biodegradable because of its low crystallinity. In periapical alveolar bone defects filled with n-TeDCPD cement, wound healing by bone reconstruction actively progresses in the early period in this examination.

In conclusion, n-TeDCPD cement induced hard tissue in dental pulp by activation of undifferentiated mesenchymal cells in dental pulp. Remodeling mechanisms of periapical bone may be induced by the low crystallinity of nTeDCPD cement.

\section{Acknowledgements}

This study was performed in the Analytical Instrument Facilities, Laboratory Animal Facilities, Biomaterial Research Facilities and Photograph-Processing Facilities, Institute of Dental Research, Osaka Dental University. This study was supported in part by a 2004 Grant-in-Aid for Scientific Research (C) from the Japan Society for the Promotion of Science (No. C:16591930).

\section{References}

[1] Linde A., The extracellular matrices of the dental pulp and dentin. J. Dent. Res., 64 (Spec Iss), pp.523-529, 1985.

[2] Tronstad L., Barbett F. \& Cervone F., Periapical bacterial plaque in teeth refractory to endodontic treatment. Endod. Dent. Traumatol., 6 (2), pp.73-77, 1990.

[3] Smith M. A. \& Steinman H.R., An in vitro evaluation of microleakage of two new and two old root canal sealers. J. Endod., 20 (1), pp. 18-21, 1994.

[4] Yoshikawa M., Terada Y. \& Toda T., Setting time and sealing ability of $\alpha$-tricalcium Phosphate cement containing titanic oxide. J. Osaka Dent. Univ., 32 (2), pp. 67-70, 1998.

[5] Koenigs J. F., Heller A. L., Brilliant J. D., Melfi R. C. \& Driskell T. D., Induced apical closure of permanent teeth in adult primates using a 
resorbable form of tricalcium phosphate ceramic. J. Endod., 1 (3), pp. 102-106, 1975.

[6] Chow L. C., Development of self-setting calcium phosphate cements. J. Ceram. Soc. Jpn., 99 (10), pp. 954-964, 1991.

[7] Hong Y. C., Wang J. T., Hong C. Y., Brown W. E. \& Chow L. C., The periapical tissue reactions to a calcium phosphate cement in the teeth of monkeys. J. Biomed. Mater. Res., 25 (4), pp. 485-498, 1991.

[8] Sarkar M.R., Wachter N., Patka P. \& Kinzl L., First histological observations on the incorporation of a novel calcium phosphate bone substitute material in human cancellous bone. J. Biomed. Mater. Res., 58 (3), pp.329-334, 2001.

[9] Sugawara A., Nishiyama M., Kusama K., Moro I., Nishimura S., Kudo I., Chow L. C. \& Takagi S., Histopathological reaction of calcium phosphate cement. Dent. Mater. J., 11 (1), pp. 11- 16, 1992.

[10] Frankenburg E. P., Goldstein S. A., Bauer T. W., Harris S. A. \& Roser R. D., Biomechanical and histological evaluation of a calcium phosphate cement. J. Bone. Joint. Surg. Am., 80 (8), pp. 1112- 1124, 1998.

[11] Yoshikawa T., Ohgushi H. \& Tamai S., Immediate bone forming capability of prefabricated osteogenic hydrioxyapatite. J. Biomed. Mater. Res., 32 (3), pp. 481- 492, 1996.

[12] Yoshikawa M., Hayami S. \& Toda T., In vivo estimation of calcium phosphate cements containing chondroitin sulfate in subcutis. Mater. Sci. Eng. C, 20 (Spec Iss), pp. 135- 141, 2002.

[13] Brown W. E. \& Chow L. C., Dental restorative cement pastes. United States Patent 1985, No.4518430, 1985.

[14] Yoshikawa M., Toda T., Mandai Y. \& Oonishi H., Hard tissue deposition In dental pulp canal by $\alpha$-tricalcium phosphate cement, Key Engineering Mater., 192-195, pp. 841- 844, 2001.

[15] Heller A. L., Koenigs J. F., Brilliant J. D., Melfi R. C. \& Driskell T. D., Direct pulp capping of permanent teeth in primates using a resorbable form of tricalcium phosphate ceramic. J. Endod., 1 (3), pp. 95- 101, 1975.

[16] Miyamoto Y., Ishikawa K., Takechi M., Toh T., Yuasa T., Nagayama M. $\&$ Suzuki, K., Histological and compositional evaluations of three types of calcium phosphate cements when implanted in subcutaneous tissue immediately after mixing. J. Biomed. Mater. Res., 48 (1), pp. 36- 42, 1999.

[17] Yoshikawa M. \& Toda T., Reconstruction of alveolar bone defect by calcium phosphate compounds, J. Biomed. Mater. Res., 53 (4), pp. 430437, 2000. 